\title{
Pump Angle and Laser Energy Dependence of Stimulated Scattering in Microcavities
}

\author{
R. Butté ${ }^{1}$ ) (a), M. Emam-Ismail (a), A. Lemaître (a), R. M. Stevenson (a), \\ M. S. Skolnick (a), D. M. WhitTaker (b), A. I. TARTAKovskit (a), \\ J. J. Baumberg (c), P. G. SAVvidis (c), and J. S. Roberts (d)
}

(a) Department of Physics and Astronomy, University of Sheffield, Sheffield S3 7RH, UK

(b) Cavendish Laboratory, University of Cambridge, Cambridge CB3 OHE, UK

(c) Department of Physics and Astronomy, University of Southampton, Southampton SO17 1BJ, UK

(d) Department of Electronic and Electrical Engineering, University of Sheffield, Sheffield S1 3JD, UK

(Received September 4, 2001; accepted September 10, 2001)

Subject classification: 42.55.Sa; 71.36.+c; 78.55.Cr; S7.12

\begin{abstract}
We show that stimulated polariton scattering in a semiconductor microcavity is observable over a wide range of laser pump angles. The ratio of idler to signal beams is found to vary strongly with angle, consistent with the expected variation of the photon fraction of the idler states, and the increasing probability of scattering of idler polaritons to the exciton reservoir with increasing wavevector. The observed variation of threshold with angle is in good agreement with a semi-classical treatment of the parametric scattering process.
\end{abstract}

Introduction Since the first observation of the strong-coupling regime (SCR) between quantum well excitons and photons in semiconductor microcavities [1], a large number of studies have been performed to understand the novel optical properties of such structures. Recently major new interest has arisen from the possibility to take advantage of the bosonic character of the cavity (exciton-)polaritons which are the normal modes of microcavities in the SCR. As a result of the quasibosonic nature of polaritons, stimulated scattering and non-linear emission were predicted to occur when occupation factors close to unity are reached [2,3]. Non-linear behaviour of the emitted photoluminescence (PL) was first observed under non-resonant excitation in both II-VI and III-V microcavities [4, 5]. Although attributed originally to final state stimulation, it was subsequently shown that this non-linear emission, at least in the III-V case, could be explained by exciton-exciton scattering alone [6,7]. Experimental evidence for stimulated scattering was first obtained in ultrafast pump-probe measurements. The weak probe pulse exhibited massive gain as a result of polariton-polariton scattering from the pump population created by resonant excitation at the point of inflection of the lower polariton branch (LPB) [8], the process being stimulated by final state occupation. Stimulated scattering for cw resonant excitation of the LPB at an angle of $16^{\circ}$ has been found recently, with very strong emission into signal and idler beams at angles of $\sim 0^{\circ}$ and $\sim 30^{\circ}$ being observed above a sharp threshold in laser power [9]. This parametric pair scattering process, in which both energy $(E)$ and momentum $(k)$ are con-

\footnotetext{
1) Corresponding author; Tel.: +44 114 2223502; Fax: +44 114 2728079; e-mail: r.butte@sheffield.ac.uk
} 
served, occurs when polariton-polariton interactions are sufficient for final state populations of the order of unity to be achieved, which in turn give rise to stimulation of the transition by final state occupation.

In the present paper, we show that stimulated polariton scattering in the $\mathrm{cw}$ regime is a very general process, with strong signal and idler beams being observed over a wide range of pump angles $\theta_{\text {pump }}$ from $6.5^{\circ}$ to $20^{\circ}$. Systematic angle-resolved PL measurements are performed to verify that the microcavity is still in the SCR above the threshold for stimulation. Above threshold, a marked blueshift of the polariton dispersion curve is found, with additional shifts to higher energy as the laser is tuned to higher energy to maintain optimum resonance conditions. We discuss how the finite width of the polariton modes, together with the renormalisation, allows resonance to be maintained over a wide range of pump angles. The threshold for stimulation is found to be nearly independent of pump angle, a result found to be in good agreement with semiclassical simulations.

Experimental Results and Discussion The experiments are performed on a $3 \lambda / 2 \mathrm{mi}$ crocavity grown by metalorganic chemical vapour deposition (MOCVD). Two sets of three $10 \mathrm{~nm}$ thick $\operatorname{In}_{0.06} \mathrm{Ga}_{0.94}$ As quantum wells are embedded at each of the antinodes of the optical field. The vacuum Rabi splitting is $\sim 6 \mathrm{meV}$. The experiments were carried out at a temperature of $\sim 14 \mathrm{~K}$ in a cold finger, wide field-of-view cryostat allowing PL measurements in transmission geometry for angles $\theta \leq \pm 60^{\circ}$. Polaritons are created resonantly at different pump angles using a tunable $\mathrm{cw} \mathrm{Ti}$ :sapphire laser. The laser beam is focused on a $50 \mu \mathrm{m}$ hole obtained by deposition of an aluminium mask on the sample, ensuring that PL is collected from a region of uniform excitation. The emission is coupled to a fibre (angular resolution of $1^{\circ}$ ), dispersed and detected by a cooled CCD. All the measurements were performed at a negative detuning $\Delta=\omega_{\text {cav }}\left(\theta=0^{\circ}\right)-\omega_{\text {exc }}=-2.7 \mathrm{meV}$.

In Figs. 1a, b, and c, PL spectra detected at $\theta=0^{\circ}$ (the angle of the signal beam, full curves) and $\theta=\theta_{\text {idler }}$ (angle of the maximum idler intensity, dashed curves) are presented for pump angles $\theta_{\text {pump }}$ of $6.5^{\circ}, 10^{\circ}$, and $15.5^{\circ}$. In each case laser powers above the threshold for stimulation are employed $\left(80,21\right.$, and $24 \mathrm{~mW}$ at $6.5^{\circ}, 10^{\circ}$, and $15.5^{\circ}$, respectively). The major result is that both signal and idler beams are observed over the wide range of $\theta_{\text {pump }}$ employed, and thus that there is no 'magic' angle for stimulated scattering, at least under the $\mathrm{cw}$ excitation conditions employed. For all pump
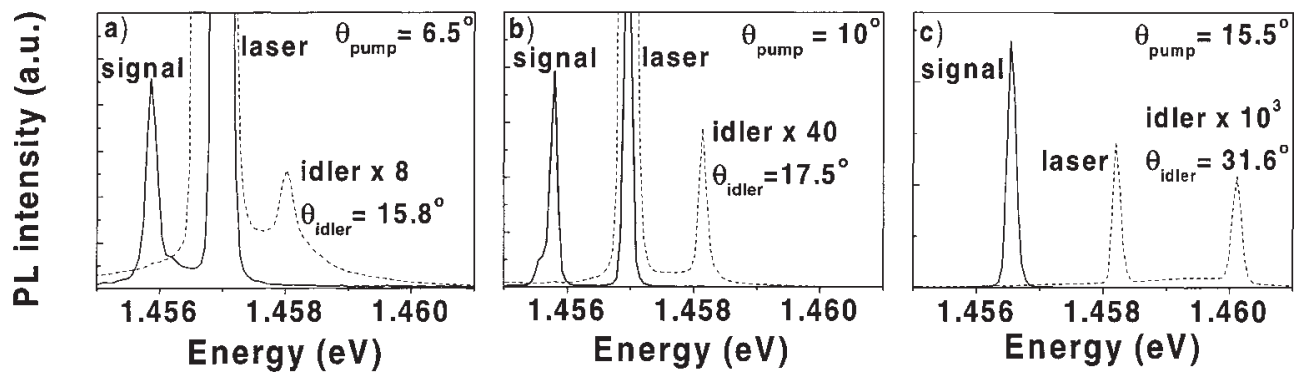

Fig. 1. Signal $\left(\theta=0^{\circ}\right.$, solid line $)$ and idler $\left(\theta=\theta_{\text {idler }}\right.$, dashed line) PL spectra for pump angles of a) $6.5^{\circ}$, b) $10^{\circ}$, and c) $15.5^{\circ}$. The idler decreases in intensity relative to the signal by $\sim 150$ from $6.5^{\circ}$ to $15.5^{\circ}$ 
angles, energy and momentum between laser, signal, and idler are conserved, consistent with the parametric nature of the process. As expected above threshold, both signal and idler spectra have very narrow linewidths with respect to the microcavity PL observed at $\theta=0^{\circ}$ and $\theta_{\text {idler }}$ below threshold, or at angles away from the signal and laser beams above threshold. In the present experiments, we measure a width of $0.03 \mathrm{meV}$ for the signal and idler, limited by the spectrometer resolution. (A double spectrometer was employed for the high resolution studies, a single spectrometer for the rest of the work presented here.) It is also noteworthy that widths as small as $0.002 \mathrm{meV}$ have been found in interferometric measurements [10]. The very narrow linewidths indicate that both the signal and idler polariton states have a long coherence time characteristic of states with macroscopic coherence, the expected signature of states with macroscopic occupancies.

At all three angles studied the idler is substantially weaker than the signal, with idler to signal intensity ratios $I_{\text {idler }} / I_{\text {signal }}$ of $0.07,0.0184$, and 0.00045 being found at $\theta_{\text {pump }}$ of $6.5^{\circ}, 10^{\circ}$, and $15.5^{\circ}$, respectively. Two main factors lead to the strongly decreasing ratio with angle: (i) the photon fraction of the idler states decreases markedly with angle $\left(0.32\right.$ at $6.5^{\circ}, 0.27$ at $10^{\circ}, 0.0043$ at $\left.15.5^{\circ}\right)$, and (ii) the increasing probability with increasing angle of scattering of the idler polaritons into high momentum states of the exciton reservoir or into localised exciton states. Correcting for the photon fractions, idler to signal populations $N_{\text {idler }} / N_{\text {signal }}$ of $0.22,0.069$, and 0.01 are found at the three angles. Thus even after correcting for the photon fractions, the $N_{\text {idler }} / N_{\text {signal }}$ ratio decreases by a factor of $\sim 20$ from $6.5^{\circ}$ to $15.5^{\circ}$, providing clear evidence for the scattering out of the idler states referred to above. The strong dependence of such scattering processes on angle arises since the energy separation between the idler and the reservoir decreases strongly with angle from $\sim 3 \mathrm{meV}$ at $6.5^{\circ}$ to $\sim 1 \mathrm{meV}$ at $15.5^{\circ}$, and thus becomes much more likely at high $k$. The scattering into reservoir states at the experimental temperature of $14 \mathrm{~K}$ also accounts for the much smaller value of $I_{\text {idler }} / I_{\text {signal }}$ of 0.00045 at $\theta_{\text {pump }}$ of $15.5^{\circ}$ found here, as compared to the value of $\sim 0.01$ reported in our earlier experiments performed at $5 \mathrm{~K}$ [9].

As in our previous work [9], it is important to verify from angle resolved PL experiments that the strong coupling limit is maintained from below to above threshold. This is demonstrated by the results for $15.5^{\circ}$ and $10^{\circ}$ shown in Figs. $2 \mathrm{a}$ and c. (Similar results are also obtained at $6.5^{\circ}$ but are not shown here.) It should be noted that for clarity, only the broad 'non-stimulated' PL peaks are plotted in Figs. $2 \mathrm{a}$ and c, with the signal and idler peaks seen in the same experiments plotted separately in Figs. $2 \mathrm{~b}$ and $\mathrm{d}$. Although shifted to higher energy with respect to the lower polariton branch measured under non-resonant excitation, the renormalised LPB PL above threshold in Figs. 2a and $\mathrm{c}$ is seen to retain its characteristic dispersion, with both the PL away from $k=0$ and the signal at $k=0$ at energies more than $1.5 \mathrm{meV}$ below that of the uncoupled cavity mode (Figs. $2 \mathrm{a}-\mathrm{d}$ ). The finite widths of the signal and idler beams, visible on the plots of Figs. $2 b$ and $d$ arise in part from Rayleigh scattering of the very intense beams, and also from the finite size of the light emitting 'condensate' regions [10, 11].

At threshold the dispersion is renormalised around $k=0$ by about $0.5 \mathrm{meV}$ to higher energy, as seen by comparison of the filled circles (non-resonantly excited PL) and the open squares (polariton PL just above threshold) in Figs. $2 \mathrm{a}$ and $\mathrm{b}$ for $\theta_{\text {pump }}=15.5^{\circ}$. This shift arises from polariton-polariton interactions, as the high occupation signal and idler states are created above threshold [12, 13]. As the laser power was increased 


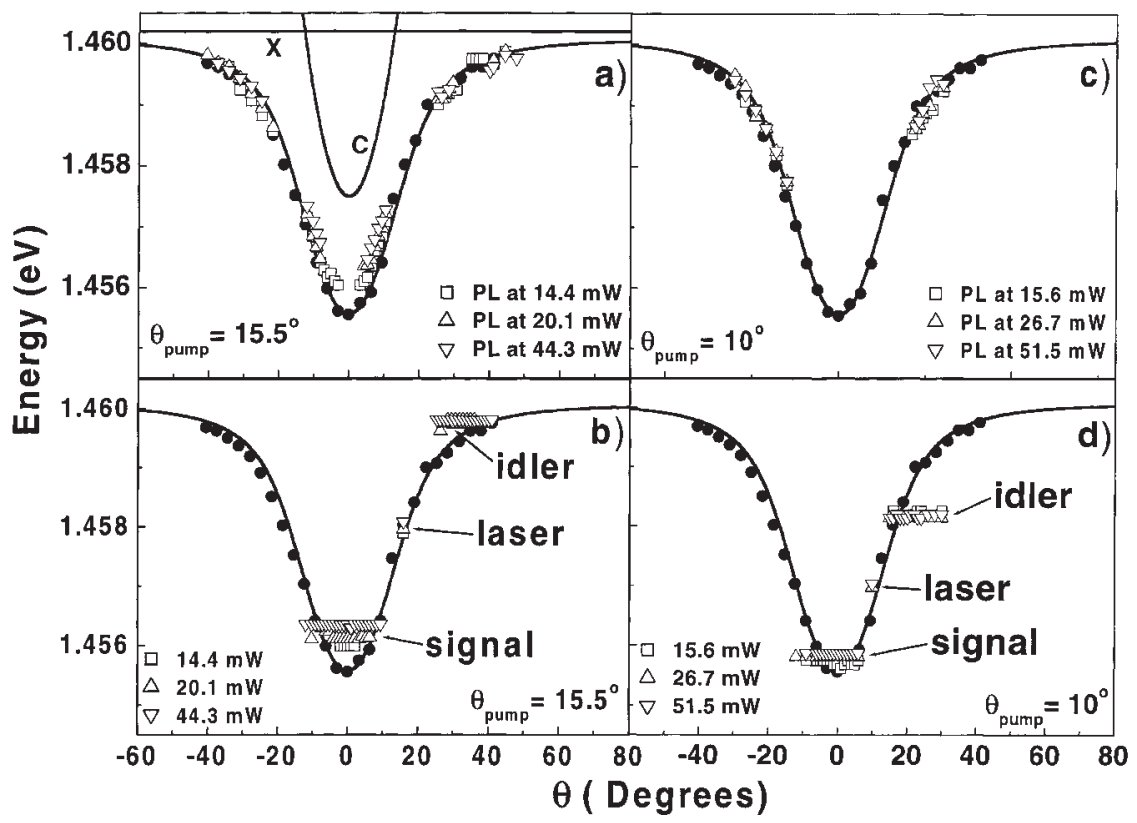

Fig. 2. a) Measured lower polariton branch dispersion in low density limit (solid circles), obtained using non-resonant excitation conditions together with fitted lower branch dispersion (solid line). Open squares and up and down triangles are lower polariton branch PL energies as a function of angle using resonant excitation $\left(\theta_{\text {pump }}=15.5^{\circ}\right)$ at three powers above threshold. Uncoupled cavity mode (C) and uncoupled exciton mode (X) are also shown. b) Same as a) but plotting signal, laser, and idler energies at the same excitation powers. c), d) Same as a) and b), respectively, for $\theta_{\text {pump }}=10^{\circ}$. The solid circles on $\mathrm{b}$ ), c), and d) represent polariton energies below threshold, as on a)

further above threshold, the laser was retuned to higher energy to maintain resonance with the renormalised dispersion curve. These additional shifts of the laser lead to additional corresponding shifts of the signal to higher energy, with accompanying much smaller shifts of the idler (of $20 \%$ of the shifts of the signal), with in all cases energy and momentum being conserved in the scattering process. It should be noted, however, that if the laser energy is kept fixed above threshold, then the signal energy also remains fixed with increasing power, as reported previously [10], although in this case lower absolute signal powers are observed due to the non-optimum coupling $[12,13]$.

The shift of the signal with laser energy for fixed laser power just above threshold is shown in Fig. 3a. A linear increase of signal energy with increasing laser energy is seen for detunings from the optimum resonance conditions of up to $\sim 0.5 \mathrm{meV}$, approximately equal to the half width of the polariton states, with a slower variation for higher detunings. These results show firstly that the signal and laser polarization fields are coupled together above threshold, as expected in a parametric process [12, 13], and secondly that near-optimum resonance can be maintained for detunings from optimum coupling of up to half the polariton linewidth (the signal intensity drops by only a factor of two over this range), as predicted in the theoretical treatments of Ciuti et al. [12] and Whittaker [13]. In addition, it is notable that not only the signal energy increases with increasing laser energy (Fig. 3b), but in addition the whole dispersion shifts to 

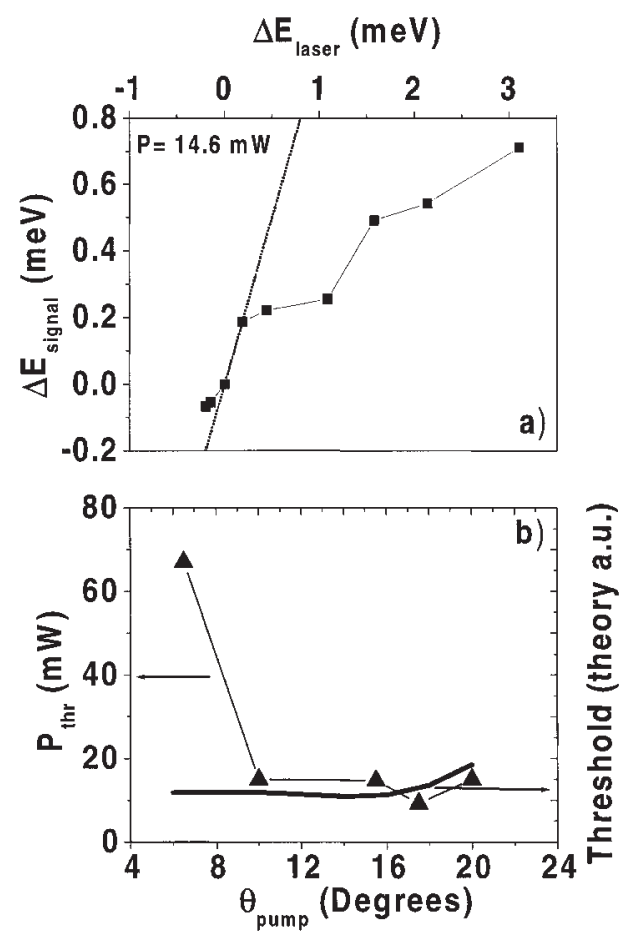

Fig. 3. a) Shift of signal energy with laser energy at fixed power above threshold. b) Threshold power versus pump angle, experimental data (solid triangles) and theoretical fit (solid line)

higher energy as seen in Fig. 2a, showing renormalisation of the whole polariton system with increasing density and laser energy, again as expected for a parametric process. Finally, it is noticeable that the renormalisation of the dispersion curve is always greater at $k=0$ than at high $k$, consistent with the much smaller shift of the idler to higher energy than that of the signal referred to earlier. This asymmetric renormalisation is not predicted theoretically, since the renormalisation arises from exciton-exciton interactions, and would be expected to have large values at high $k$.

Further theoretical treatment is required to understand this point.

We have shown so far in Figs. 1 and 2 that stimulated scattering can be observed over a wide range of pump angles. We show in Fig. $3 b$ the variation of the threshold power for stimulation over the range of angles studied from $6.5^{\circ}$ to $20^{\circ}$. (Above $15.5^{\circ}$ the idler becomes unobservably weak, and so results were not presented in Figs. 1 and 2.) It is seen that the threshold is almost independent of $\theta_{\text {pump }}$ over a wide range of pump angles from $10^{\circ}$ to $20^{\circ}$, with a marked increase only being observed at low angle. This overall behaviour is reproduced well by a semi-classical treatment of the parametric process in the $\mathrm{cw}$ regime, as shown in Fig. 3b, where a non-linear exciton is strongly coupled to a cavity mode, as described in Ref. [13]. The near independence of threshold on $\theta_{\text {pump }}$ arises since energy and wavevector can still be conserved for differing $\theta_{\text {pump }}$ when allowance is made for the finite polariton linewidths. Further details of the theory will be described elsewhere. Disagreement between experiment and theory is found only at low angle. This discrepancy may arise from the finite angular width of the pump beam in the experiment, the angular spread having a larger fractional effect at small angle and thus causing a marked increase in threshold in experiment relative to theory.

Conclusion In conclusion, we have reported the observation of stimulated polariton scattering under resonant excitation over a wide range of pump angles. The ratio of idler to signal beams was shown to be controlled by the variation of the photon fraction with angle, and the probability of scattering of the idler polaritons to the exciton reservoir at high wavevector or to localised exciton states. The threshold power for 
stimulation was found to be almost independent of $\theta_{\text {pump }}$ for a wide range of pump angles, in agreement with theory predictions.

Acknowledgements R. Butté and P. G. Savvidis acknowledge the financial support provided through the European Community's Human Potential Programme under contract HPRN-CT-1999-00132, CLERMONT.

\section{References}

[1] C. Weisbuch et al., Phys. Rev. Lett. 69, 3314 (1992).

[2] A. ImamoĞLu et al., Phys. Rev. A 53, 4250 (1996).

[3] F. Tassone and Y. Yamamoto, Phys. Rev. B 59, 10830 (1999).

[4] Le Si Dang et al., Phys. Rev. Lett. 81, 3920 (1998).

[5] P. Senellart and J. Bloch, Phys. Rev. Lett. 82, 1233 (1999).

[6] A. I. Tartakovskil et al., Phys. Rev. B 62, R2283 (2000).

[7] P. Senellart et al., Phys. Rev. B 62, R16263 (2000).

[8] P. G. SAvvidis et al., Phys. Rev. Lett. 84, 1547 (2000).

[9] R. M. Stevenson et al., Phys. Rev. Lett. 85, 3680 (2000).

[10] J. J. BAumberg et al., Phys. Rev. B 62, R13278 (2000).

[11] R. Houdré et al., Phys. Rev. Lett. 85, 2793 (2000); Physica E 7, 625 (2000).

[12] C. Ciuti et al., Phys. Rev. B 63, 041303 (2000).

[13] D. M. Whittaker, Phys. Rev. B 63, 193305 (2001). 